\title{
Recurrent lactic acidosis and hypoglycemia with inadvertent metformin use: a case of look-alike pills
}

\author{
Tess Jacob1, Renee Garrick² and Michael D Goldberg' \\ Divisions of ${ }^{1}$ Endocrinology, and ${ }^{2}$ Nephrology, Department of Medicine, Westchester Medical Center , Valhalla, \\ New York, USA \\ Correspondence \\ should be addressed \\ to M D Goldberg \\ Email \\ Michael.Goldberg@ \\ wmchealth.org
}

\section{Summary}

Metformin is recommended as the first-line agent for the treatment of type 2 diabetes. Although this drug has a generally good safety profile, rare but potentially serious adverse effects may occur. Metformin-associated lactic acidosis, although very uncommon, carries a significant risk of mortality. The relationship between metformin accumulation and lactic acidosis is complex and is affected by the presence of comorbid conditions such as renal and hepatic disease. Plasma metformin levels do not reliably correlate with the severity of lactic acidosis. We present a case of inadvertent metformin overdose in a patient with both renal failure and hepatic cirrhosis, leading to two episodes of lactic acidosis and hypoglycemia. The patient was successfully treated with hemodialysis both times and did not develop any further lactic acidosis or hypoglycemia, after the identification of metformin tablets accidentally mixed in with his supply of sevelamer tablets. Early initiation of renal replacement therapy is key in decreasing lactic acidosis-associated mortality.

\section{Learning points:}

- When a toxic ingestion is suspected, direct visualization of the patient's pills is advised in order to rule out the possibility of patient- or pharmacist-related medication errors.

- Though sending a specimen for determination of the plasma metformin concentration is important when a metformin-treated patient with diabetes presents with lactic acidosis, complex relationships exist between metformin accumulation, hyperlactatemia and acidosis, and the drug may not always be the precipitating factor.

- Intermittent hemodialysis is recommended as the first-line treatment for metformin-associated lactic acidosis (MALA).

- An investigational delayed-release form of metformin with reduced systemic absorption may carry a lower risk for MALA in patients with renal insufficiency, in whom metformin therapy may presently be contraindicated.

\section{Background}

Metformin is currently recommended by many professional organizations as the first-line agent for the treatment of type 2 diabetes (1). Its multiple advantages include potent glucose-lowering efficacy, absence of weight gain, minimal risk of hypoglycemia and low cost. In the United Kingdom Prospective Diabetes Study (UKPDS), analysis of a subset of overweight patients demonstrated significant risk reductions for diabetes-related vascular endpoints, diabetes-related death and all-cause mortality in patients treated with metformin vs those receiving conventional therapy with diet alone (2). Metformin decreases hepatic glucose production by suppressing gluconeogenesis via inhibition of mitochondrial oxidative phosphorylation (3). Metformin also decreases insulin 
resistance, by enhancing peripheral insulin-mediated glucose uptake (3). Under typical conditions, treatment with metformin may cause small, clinically insignificant increases in blood lactate concentration (4). However, in the presence of reduced metformin clearance due to impaired renal function, reduced lactate clearance due to impaired hepatic function and/or comorbid conditions that predispose to hypoxemia or hypoperfusion, there is an increased risk for clinically significant metforminassociated lactic acidosis (MALA) (5).

\section{Case presentation}

A 58-year-old man presented to the emergency department with progressively severe nausea, diarrhea, dizziness and lethargy of one week's duration. On the day of admission, he experienced 12-13 episodes of watery diarrhea and was not able to tolerate any oral intake. His medical history included type 2 diabetes, hepatic cirrhosis due to chronic hepatitis $C$ virus infection and end-stage renal disease, which had developed rapidly two months prior to presentation and was felt to be due to hepatorenal syndrome. His Model for End-Stage Liver Disease (MELD) score was 28 , and he had experienced recurrent ascites as well as upper gastrointestinal bleeding due to esophageal varices. He was being managed with thrice weekly hemodialysis and had been listed for a dual kidney and liver transplant. His last hemodialysis session was two days prior to presentation. His medications included glimepiride $0.5 \mathrm{mg}$ daily, lactulose, rifaximin, sevelamer, pantoprazole and ergocalciferol. His diabetes had previously been treated with metformin and dapagliflozin, but these had been discontinued two months prior, when his renal function had worsened. Blood pressure was $151 / 73 \mathrm{mmHg}$, heart rate was $125 \mathrm{bpm}$ and temperature was $96.8^{\circ} \mathrm{F}$. He was somnolent, and ascites was present on examination.

\section{Investigation}

Laboratory evaluation revealed severe hypoglycemia (glucose: $14 \mathrm{mg} / \mathrm{dL}$ ), severe metabolic acidosis (pH: 6.98, serum carbon dioxide: $7 \mathrm{mEq} / \mathrm{L}$ ) and an elevated lactate level of $>20.1 \mathrm{mmol} / \mathrm{L}$. Blood urea nitrogen was $38 \mathrm{mg} / \mathrm{dL}$ and serum creatinine was $6.5 \mathrm{mg} / \mathrm{dL}$. Serum total bilirubin and albumin were within normal limits, and prothrombin time was $12.3 \mathrm{~s}$ (normal<12.0). Computed tomography of the abdomen revealed a cirrhotic liver, large ascites and stable partially occlusive thrombus of the portal vein, with no evidence for bowel ischemia.
The patient's wife, who organized his medications at home, brought in his pill bottles for review. None of them were labeled as metformin, and she was certain that she had discarded the metformin pills several weeks before.

\section{Treatment}

The lactic acidosis resolved with intermittent hemodialysis, and after initial correction of the hypoglycemia, the blood glucose levels remained stable in the $180-250 \mathrm{mg} /$ $\mathrm{dL}$ range. Large-volume paracentesis was performed. The patient's mental status improved, and he was able to tolerate full oral intake. Blood cultures drawn on admission remained negative. The patient was discharged home in stable condition after four days, with plans to resume his usual outpatient hemodialysis sessions and follow-up in the endocrinology clinic. Glimepiride had not been administered as an inpatient, nor was it resumed on discharge.

Approximately $36 \mathrm{~h}$ later, the patient returned to the emergency department with recurrent nausea, diarrhea, dizziness and lethargy. Laboratory evaluation revealed severe hypoglycemia (glucose: $30 \mathrm{mg} / \mathrm{dL}$ ), severe metabolic acidosis (pH: 6.96, serum carbon dioxide $<6 \mathrm{mEq} / \mathrm{L}$ ) and an elevated lactate level of $20.3 \mathrm{mmol} / \mathrm{L}$. Treatment was re-initiated with hemodialysis, and the hypoglycemia was corrected.

This time on admission, the patient's pill bottles were opened and the contents were examined. On close inspection, the bottle labeled Renvela (sevelamer) was noted to contain two groups of similar-appearing oblong white tablets, that were slightly different sizes and had different imprints (Fig. 1). Analysis of the imprints confirmed that the bottle contained a mixture of sevelamer $800 \mathrm{mg}$ tablets and metformin $1000 \mathrm{mg}$ tablets, the latter making up the majority. The patient had been following the dosing instructions for sevelamer, taking two tablets from this bottle three times daily with meals. For convenience, his wife had recently refilled the contents of this bottle from a larger bottle of pills that she assumed were also sevelamer.

A plasma metformin level that had been sent from the blood specimen obtained during the second emergency department visit, later returned elevated at $33 \mu \mathrm{g} / \mathrm{mL}$ (therapeutic range: $1-2 \mu \mathrm{g} / \mathrm{mL}$ ).

\section{Outcome and follow-up}

The patient was discharged home in stable condition after three days. Five months later, his diabetes is being 


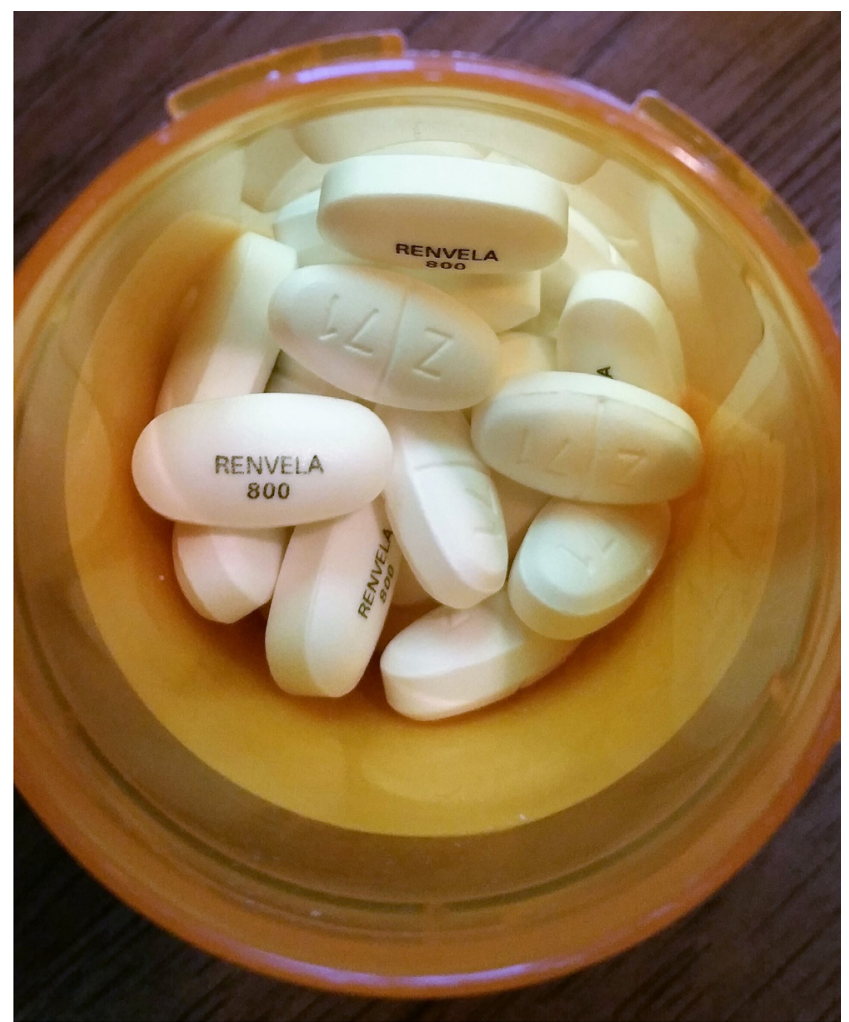

Figure 1

Look-alike pills. The Z/71 imprint indicates metformin $1000 \mathrm{mg}$ tablets mixed in with Renvela (sevelamer) tablets.

managed with insulin monotherapy, and there has been no recurrent hypoglycemia or lactic acidosis. He has undergone a transjugular intrahepatic portocaval shunt (TIPS) and continues to await a dual kidney and liver transplant.

\section{Discussion}

Our patient presented with severe lactic acidosis, the etiology of which was initially unclear. Inappropriate continued use of metformin was considered, but this possibility was ruled out after the patient's pill bottle labels were reviewed. In the absence of sepsis or toxic ingestion, the presumed cause was voluminous diarrhea on the background of end-stage renal disease and cirrhosis. However, when the actual contents of the patient's pill bottles were scrutinized after his readmission with a second episode of lactic acidosis, and it was determined that he had been taking up to $6000 \mathrm{mg}$ of metformin daily, MALA was felt to be the true diagnosis.

MALA is traditionally defined as lactic acidosis (elevated anion gap metabolic acidosis with $\mathrm{pH}<7.35$ and lactate $>5 \mathrm{mmol} / \mathrm{L}$ ) occurring in a metformin- treated patient (5). Lactic acidosis can present with anorexia, nausea, vomiting and abdominal pain; more severe cases can be marked by hypotension, respiratory failure, arrhythmia and hypothermia (6). A high index of suspicion is needed in diabetic individuals presenting with symptoms suggestive of lactic acidosis, as the mortality rate in MALA may be as high as 50\% (7).

The diagnosis of MALA is often challenging given its nonspecific signs and symptoms, as well as the fact that measurement of plasma metformin levels is not always readily available. A study by Kajbaf and Lalau, reviewing the pharmacovigilance reporting of cases of MALA worldwide, found that fewer than half met appropriate diagnostic criteria for lactic acidosis, and in only $14 \%$ was plasma metformin measured (8). The true incidence of MALA is debated in the literature. Population-based studies have estimated a rate of 2-9 cases of lactic acidosis in metformin users per 100000 person years (9); the rate of lactic acidosis in non-metformin users may actually be similar (4). A systematic review of 176 studies by Salpeter and coworkers revealed no cases of lactic acidosis in both metformin users and non-metformin users (9).

The relationship between metformin accumulation and lactic acidosis is complex (5). Not all patients with isolated metformin overdose develop lactic acidosis or even significant hyperlactatemia $(5,10)$. Plasma metformin concentrations considerably higher than the $33 \mu \mathrm{g} / \mathrm{mL}$ measured in this case have been documented - sometimes without concomitant lactic acidosis (5) though it is possible that the value may have been higher if measured at the time of the first admission. There also appears to be no correlation between plasma metformin levels and mortality (11). It has been suggested that the measurement of metformin levels in erythrocytes may provide a reliable indicator of tissue accumulation, as the estimated half-life for the elimination of the drug from erythrocytes $(23.4 \mathrm{~h})$ exceeds that in plasma $(2.7 \mathrm{~h})(12)$.

Some authors have suggested a new classification of lactic acidosis in metformin-treated patients, with the following diagnostic categories: 'metformin-unrelated lactic acidosis (MULA)' (low metformin concentration, other triggers for lactic acidosis present), 'metformininduced lactic acidosis (MILA)' (exclusively due to metformin), 'metformin-associated lactic acidosis (MALA)' (both metformin accumulation and systemic diseases contributing to hyperlactatemia) and 'lactic acidosis in metformin therapy (LAMT)' (metformin concentration not available) (10). Within this scheme, our patient would be best classified as having MILA. Having said that, the proposed new terminology has not yet been widely 
adapted, and the general designation 'MALA' is retained in this discussion to mirror its use in the referenced literature.

Renal failure and hepatic failure are known risk factors for MALA. With impaired clearance of metformin in renal failure, and impaired clearance of lactate in hepatic failure, the presence of these comorbidities may increase an individual's risk of lactic acidosis with metformin use (3). Our patient was therefore at particularly high risk for this rare complication. Though not present in our patient, other risk factors for lactic acidosis include those in which lactate production is increased, such as hypotension, ischemia and sepsis (5).

The initial management of MALA includes resuscitation and supportive care, and in the setting of acute metformin overdose, there may be a role for gastrointestinal decontamination to prevent further drug absorption. Beyond these interventions, extracorporeal treatments (ECTRs) are key in the treatment of MALA (13). Conventional dialysis modalities are generally successful in clearing metformin owing to the drug's low molecular weight and limited protein binding (6). Based on a systematic review of 175 studies, the Extracorporeal Treatments in Poisoning Workgroup has recommended ECTR in the treatment of MALA, citing multiple potential advantages over bicarbonate therapy (13). In addition, guidelines were set forth regarding its initiation: ECTR was recommended if the lactate level is $>20 \mathrm{mmol} / \mathrm{L}$, the blood $\mathrm{pH}$ is $\leq 7.0$ or if standard therapy with supportive care and bicarbonate fails; ECTR was suggested if the lactate level is $15-20 \mathrm{mmol} / \mathrm{L}$ or the blood $\mathrm{pH}$ is $7.0-7.1$. The presence of comorbidities, such as shock, impaired kidney function, liver failure and decreased level of consciousness, should lower these thresholds for treatment (13). A specific plasma metformin concentration was not specified as a criterion for ECTR (13). Intermittent hemodialysis (HD) was recommended as the first-line initial ECTR, with continuous renal replacement therapy (CRRT) felt to be an acceptable alternative if hemodialysis is not available; if subsequent treatment is necessary, either of the two modalities may be used (13). The recommended criteria for cessation of ECTR were a lactate level $<3 \mathrm{mmol} / \mathrm{L}$ and a blood $\mathrm{pH}>7.35$; a specific metformin concentration target was not recommended (13). Of note, an extended duration of hemodialysis or repeated sessions, may be required to prevent 'rebound' lactic acidosis, a phenomenon that may result from the redistribution of metformin from deep compartments into the intravascular space $(6,13)$. In fact, it is possible that in this case, the recurrent lactic acidosis may have at least partially reflected such a 'rebound' phenomenon, especially as only a relatively limited amount of additional metformin could have been ingested in the short interval between presentations. This could further reconcile the absence of a more significantly elevated plasma metformin concentration at the time of readmission. A recent pharmacokinetic analysis of 12 cases of MALA demonstrated a prolonged elimination of accumulated metformin, even after dialysis therapy; the estimated mean terminal half-life in plasma was $51.9 \mathrm{~h}$, and the drug remained detectable for up to 13 days (12).

A new formulation of delayed-release (DR) metformin is under investigation, which delivers the drug primarily to the ileum, where systemic absorption is very low. Clinical studies have demonstrated that, compared with immediate-release (IR) and extended-release (XR) metformin, the DR formulation results in approximately $50 \%$ lower plasma metformin levels (14). Moreover, the DR formulation was shown to be more potent than the $\mathrm{XR}$ formulation in terms of glucose-lowering efficacy (14). Notably, metformin DR resulted in no increase in plasma lactate levels compared with placebo, which may theoretically translate to a lower risk for MALA in patients with reduced metformin clearance due to renal insufficiency (14).

Our patient's two episodes of lactic acidosis were accompanied by severe hypoglycemia. While, at the time of the first admission, this was felt most likely related to the effect of the sulfonylurea in the setting of renal and hepatic failure and poor oral intake, the fact that it recurred after hemodialysis and cessation of glimepiride treatment prompted consideration of metformin intoxication as a contributing factor. Hypoglycemia is rare in patients taking metformin, but the risk may be increased in the context of poor caloric intake (15). Impaired hepatic and renal gluconeogenesis likely compounded the risk in this patient's case.

In conclusion, we present a case of recurrent MALA that resulted from inadvertent overdose in a high-risk patient. MALA can be a challenging diagnosis, and renal replacement therapy is the mainstay of treatment. The investigational DR formulation of metformin may prove to carry a lower risk for MALA, thus potentially expanding the safe use of this potent glucose-lowering medication to populations for which it is currently contraindicated. Our experience with this patient underscored the importance of direct visualization of all medications when a toxic ingestion is on the differential diagnosis and served as a reminder that patients may compound the risk for medication errors when they transfer pills from one bottle to another. 


\section{Declaration of interest}

The authors declare that there is no conflict of interest that could be perceived as prejudicing the impartiality of the research reported.

\section{Funding}

This research did not receive any specific grant from any funding agency in the public, commercial, or not-for-profit sector.

\section{Patient consent}

Written informed consent for publication of their clinical details and clinical images was obtained from the patient.

\section{Author contribution statement}

T Jacob, R Garrick and M D Goldberg were involved in the care of the patient; $T$ Jacob performed the literature review and drafted the manuscript; R Garrick and M D Goldberg critically reviewed and edited the manuscript.

\section{References}

1 Inzucchi SE, Bergenstal RM, Buse JB, Diamant M, Ferrannini E, Nauck M, Peters AL, Tsapas A, Wender R \& Matthews DR. Management of hyperglycemia in type 2 diabetes, 2015: a patientcentered approach. Update to a position statement of the American Diabetes Association and the European Association for the Study of Diabetes. Diabetes Care 201538 140-149. (https://doi.org/10.2337/ dc14-2441)

2 Effect of intensive blood-glucose control with metformin on complications in overweight patients with type 2 diabetes (UKPDS 34). UK prospective diabetes study (UKPDS) group. Lancet 1998352 854-865.

3 Bailey CJ \& Turner RC. Metformin. New England Journal of Medicine 1996334 574-579. (https://doi.org/10.1056/ NEJM199602293340906)

4 Inzucchi SE, Lipska KJ, Mayo H, Bailey CJ \& McGuire DK. Metformin in patients with type 2 diabetes and kidney disease: a systematic review. JAMA 2014312 2668-2675. (https://doi.org/10.1001/ jama.2014.15298)
5 Lalau JD \& Race JM. Metformin and lactic acidosis in diabetic humans. Diabetes, Obesity and Metabolism 20002 131-137. (https:// doi.org/10.1046/j.1463-1326.2000.00053.x)

6 Prikis M, Mesler EL, Hood VL \& Weise WJ. When a friend can become an enemy! Recognition and management of metforminassociated lactic acidosis. Kidney International 200772 1157-1160. (https://doi.org/10.1038/sj.ki.5002346)

7 Kajbaf F \& Lalau JD. Mortality rate in so-called 'metforminassociated lactic acidosis': a review of the data since the 1960s. Pharmacoepidemiology and Drug Safety 201423 1123-1127. (https:// doi.org/10.1002/pds.3689)

8 Kajbaf F \& Lalau JD. The criteria for metformin-associated lactic acidosis: the quality of reporting in a large pharmacovigilance database. Diabetic Medicine 201330 345-348. (https://doi. org/10.1111/dme.12017)

9 Salpeter SR, Greyber E, Pasternak GA \& Salpeter EE. Risk of fatal and nonfatal lactic acidosis with metformin use in type 2 diabetes mellitus. Cochrane Database of Systematic Reviews 20104 CD002967. (https://doi.org/10.1002/14651858.CD002967.pub4)

10 Lalau JD, Kajbaf F, Protti A, Christensen MM, Broe ME \& Wiernsperger N. Metformin-associated lactic acidosis (MALA): moving towards a new paradigm. Diabetes, Obesity and Metabolism 201719 1502-1512. (https://doi.org/10.1111/dom.12974)

11 Lalau JD \& Race JM. Lactic acidosis in metformin-treated patients. prognostic value of arterial lactate levels and plasma metformin concentrations. Drug Safety 199920 377-384. (https://doi. org/10.2165/00002018-199920040-00006)

12 Kajbaf F, Bennis Y, Hurtel-Lemaire AS, Andrejak M \& Lalau JD. Unexpectedly long half-life of metformin elimination in cases of metformin accumulation. Diabetic Medicine 201633 105-110. (https://doi.org/10.1111/dme.12959)

13 Calello DP, Liu KD, Wiegand TJ, Roberts DM, Lavergne V, Gosselin S, Hoffman RS, Nolin TD, Ghannoum M \& Extracorporeal Treatments in Poisoning Workgroup. Extracorporeal treatment for metformin poisoning: systematic review and recommendations from the extracorporeal treatments in poisoning workgroup. Critical Care Medicine 201543 1716-1730. (https://doi.org/10.1097/ CCM.0000000000001002)

14 Buse JB, DeFronzo RA, Rosenstock J, Kim T, Burns C, Skare S, Baron A $\&$ Fineman $\mathrm{M}$. The primary glucose-lowering effect of metformin resides in the gut, not the circulation: results from short-term pharmacokinetic and 12-week dose-ranging studies. Diabetes Care 201639 198-205. (https://doi.org/10.2337/dc16-1340)

15 Inkster B, Zammitt NN \& Frier BM. Drug-induced hypoglycemia in type 2 diabetes. Expert Opinion on Drug Safety 201211 597-614. (https://doi.org/10.1517/14740338.2012.694424)

Received in final form 21 November 2017 Accepted 30 November 2017 